\title{
Diseases of the Chest Wall, Pleura, and Diaphragm
}

\author{
Aine M. Kelly and Thomas Frauenfelder
}

\section{Learning Objectives}

- To learn which imaging modalities to use in evaluating the chest wall, pleura, and diaphragm.

- To generate a differential diagnosis for diseases of the chest wall, pleura, and diaphragm.

- To distinguish extrapulmonary pathology from pulmonary pathology on imaging studies.

- To apply physics principles when assessing fluid and air in the pleural space.

- To become familiar with the imaging appearances in diaphragmatic trauma.

\subsection{Introduction}

When imaging the chest wall, pleura, and diaphragm, chest radiography is helpful in problem-solving, whereas in the mediastinum, CT is much more useful. With extrapulmonary lesions arising in the superior sulcus (apical) region, or chest wall masses, magnetic resonance imaging (MRI) is excellent because of its tissue characterization abilities.

In general, normal variants, unlike disease, are reasonably symmetric and often bilateral, and thus a side-by-side comparison can be made. The soft tissues, fat, and musculature should be fairly symmetric. When assessing for skeletal abnormalities, which are frequently challenging to see, it is critical to examine the individual bones carefully, one by one. To help differentiate an extrapulmonary (chest wall,

\footnotetext{
A. M. Kelly $(\bowtie)$

Department of Radiology, University of Michigan,

Ann Arbor, MI, USA

e-mail: ainekell@med.umich.edu

T. Frauenfelder

Institute of diagnostic and interventional Radiology, University

Hospital Zurich, Zurich, Switzerland

e-mail: Thomas.frauenfelder@usz.ch
}

pleural, or diaphragmatic) lesion from a pulmonary parenchymal lesion, assessing the angle the mass makes with the lung edge (obtuse angle for extra-parenchymal masses and acute angles for pulmonary masses) may be helpful. With extrapulmonary masses, it can be difficult to determine if it is arising from the chest wall or pleura, as their shapes may be similar, but the presence of bone destruction suggests an extrapleural origin.

\subsection{Chest Wall Disease}

The chest wall should normally be symmetric with the most common causes of apparent asymmetry being patient rotation or thoracic kyphoscoliosis. The more lucent side is that to which the patient is rotated toward (e.g., in a right anterior oblique (RAO)/left posterior oblique (LPO) position, the left side becomes more lucent). Chest radiography is useful to detect subcutaneous emphysema and calcification and to evaluate skeletal disease.

Key Point

- Normal variants tend to be symmetric.

\subsection{Hyperlucent or Hyperopaque Hemithorax}

Asymmetry in the opacity (hyperlucency or hyper-opacity) of the hemithoraces has many causes, and a reliable approach (once rotation has been excluded) is to start from the outside (skin) and work inward (through subcutaneous tissues, muscles, bones, pleura, and pulmonary parenchyma), to reach the vasculature (pulmonary arteries). Tables 9.1 and 9.2 depict the most common causes of a hyperlucent or hyperopaque hemithorax. 


\section{Table 9.1 Causes of a hyperlucent hemithorax}

Rotation to that side - patient positioning or thoracic kyphoscoliosis Mastectomy or chest wall resection on the ipsilateral side

Poland syndrome - congenital

Stroke/polio - acquired disease

Subcutaneous emphysema (e.g., from trauma)

Pneumothorax

Emphysema

Hypoplastic lung

Pulmonary artery hypoplasia

Table 9.2 Causes of a hyperopaque hemithorax

Rotation away from that side-patient positioning or thoracic kyphoscoliosis

Chest wall mass on the ipsilateral side

Pleural effusion-large

Fibrothorax

Consolidation involving most of the ipsilateral lung

Mass-large, in ipsilateral lung

Pneumonectomy or lobectomy on the contralateral side

\section{Key Point}

- With unequal lucency of the hemithoraces, it is helpful to start by working one's way in from the outside (skin and subcutaneous tissues) to the inside (pulmonary vasculature) to evaluate the cause. Don't forget patient rotation and kyphoscoliosis!

\subsection{Soft Tissue Masses}

These need to be differentiated from asymmetries due to patient positioning, contralateral surgery (such as mastectomy), and contralateral atrophy (e.g., poliomyelitis or stroke). Lipomas are the most common benign primary tumor, and the patient can often provide a history of a stable or slowly enlarging soft mass. Other benign entities include hemangiomas, neurogenic lesions (neurofibromas, schwannomas), and fibromatosis. Neurofibromas arise from intercostal nerves or paraspinal ganglia which can be multiple in von Recklinghausen disease, neurofibromatosis type 1 . The most common nonneoplastic tumor of the thoracic skeleton is fibrous dysplasia, accounting for $30 \%$ of chest wall benign bone tumors [1]. These slow-growing tumors arise in the posterolateral ribs and are usually asymptomatic until pathologic fractures or pressure symptoms occur. On imaging, fibrous dysplasia presents as an expansile lytic lesion with a "ground glass" or hazy or matrix (Fig. 9.1).

Chest wall malignancies are rare and can result from direct spread of or metastatic disease from adjacent
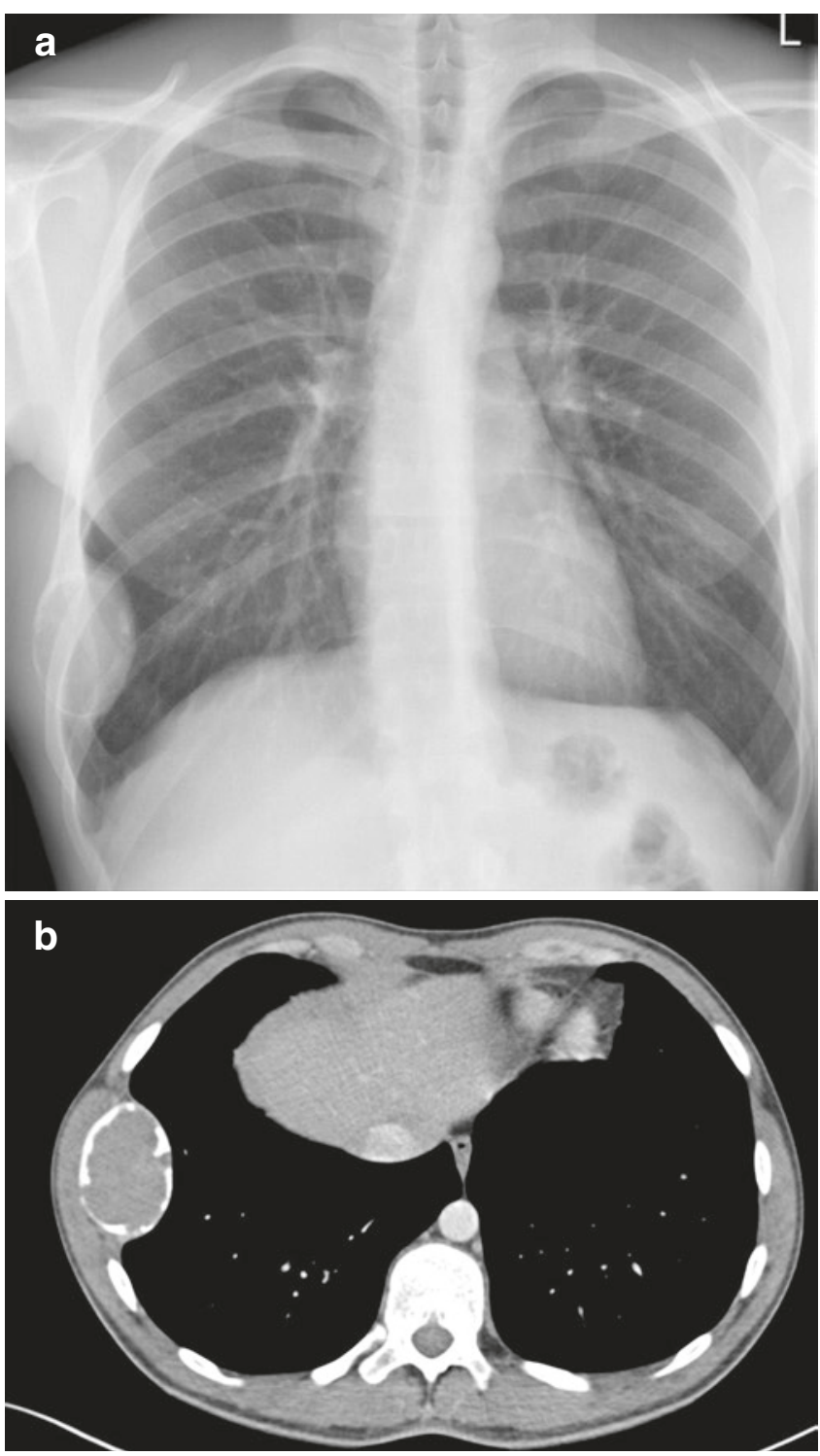

Fig. 9.1 (a, b). A 25-year-old male with a palpable hard mass in the right lateral chest wall. Frontal chest radiography (a) shows an expansile oval lesion of the right eighth rib laterally, with a hazy matrix. Axial $\mathrm{CT}$ demonstrates an expansile lesion of the lateral right rib with welldefined borders

tumors including primary bronchogenic neoplasms, mesothelioma, thymoma, and breast cancer. Plasmacytoma and multiple myeloma are the most common malignant neoplasms of the thoracic skeleton and can present as extrapulmonary masses with bone destruction, similar appearances to other metastases [2]. Well-defined bone lesions with a punched-out appearance are found, which can erode, expand, and destroy cortex, sometimes with surrounding bone reaction, the so-called soap bubble appearance. Other primary chest wall malignant bone tumors are rare and include chondrosarcoma, osteosarcoma, Ewing's sarcoma family of tumors, (evaluate for 
chondroid or osseous matrix here), multiple myeloma/ plasmacytoma, fibrosarcoma, and malignant fibrohisticytoma in adults. Malignant soft tissue masses are a heterogenous group and include undifferentiated pleomorphic sarcoma, liposarcoma, angiosarcoma, and malignant peripheral nerve sheath tumor (which result from malignant transformation of a peripheral chest wall neurofibroma in neurofibromatosis 1 patients) [3-6].

SAPHO (synovitis, acne, pustulosis, hyperostosis, and osteitis) syndrome is a distinct clinical entity representing involvement of the musculoskeletal and dermatologic systems. SAPHO syndrome can mimic some of the more common disease entities such as infection, tumor, and other inflammatory arthropathies. Treatment is empirical, including nonsteroidal anti-inflammatory drugs (NSAIDs) as firstline agents, as well as corticosteroids, disease-modifying antirheumatic drugs, antibiotics, and biological agents. Imaging can be helpful by offering a detailed evaluation of the abnormalities [7]. Ossification of the costoclavicular ligament and hyperostosis at the sternal end of the first ribs are important early findings.

\subsection{Inflammatory Disease of the Chest Wall}

Chest wall involvement with bacterial infection typically involves ribs and sternum but is rare. Hematogenous or direct spread from a nearby infectious process can cause osteomyelitis with Staphylococcus aureus or Pseudomonas aeruginosa most commonly implicated. Soft tissue swelling and underlying rib destruction with periosteal reaction may be seen by radiography in chronic cases of osteomyelitis; however, cross-sectional imaging with CT and MRI is much more sensitive and specific. MRI is more sensitive than CT in detecting early osteomyelitis manifest as marrow hypointensity on $\mathrm{T} 1$ and bone marrow edema on T2 images. On the other hand, CT better depicts cortical destruction and periosteal reaction compared with MRI.

Chest wall involvement with tuberculosis (TB) is uncommon, with hematogenous spread or, very rarely, direct extension from the adjacent pleural space (empyema necessitans) or the underlying lung parenchyma. Chest wall involvement with TB manifests as soft tissue masses with calcification and rim enhancement, and osseous and cartilaginous destruction can be seen [8]. In immunocompromised patients, fungal infections of the chest wall and bones can occur with Aspergillus species accounting for $80-90 \%$ of cases [9]. Thoracic actinomycosis can also invade the chest wall, creating fistulous tracts and empyema necessitatis. In post-op patients, nocardia infection can also cause destructive chest wall masses.

\subsection{Calcification}

Soft tissue calcification may be metastatic or dystrophic. Metastatic calcification is found with elevated serum calcium with calcium hydroxyapatite crystals being deposited in multiple locations, including the lung, stomach, kidney, and vascular system [10]. Dystrophic calcification occurs in altered, necrotic, or dead tissues, in patients with normal serum calcium and phosphorus levels, for example, in connective tissue disorders, like dermatomyositis, where it can be deposited in the subcutaneous tissues, muscles, and fascial planes as calcification universalis.

\subsubsection{Pectus Excavatum}

In this congenital variant of chest wall development (incidence $1 / 400-1 / 1000$ ), the sternum is depressed relative to the anterior ribs and often tilts rightward, with the mediastinal structures displaced leftward $[9,11]$. Many cases are asymptomatic and cosmetic only, but if severe [9].

\subsubsection{Injuries to the Thoracic Skeleton/Rib Fractures/Trauma}

Fractures of the upper ribs and scapulae suggest severe trauma and should prompt a search for associated injury to the aorta, great vessels, and brachial plexus [12]. Fractures of the sternum and medial clavicle have higher risks of associated vascular or cardiac injury. Sternal fractures are extremely difficult to see on frontal chest radiographs but may be seen on the lateral view. CT is indicated if they are suspected. Nearly all sternal fractures will be visible on CT, and adjacent hematoma can be demonstrated. With sternal fractures, one should evaluate for associated cardiac or vascular injury. For suspected fractures of the lower ribs, abdominal radiographs are more helpful, as they are better penetrated. Fractures of the lower ribs may be associated with hepatic, splenic, or renal injury, and abdominal CT is indicated in these cases.

A flail chest (or flail segment) occurs when there are five or more consecutive rib fractures (or three or more ribs are fractured in two or more places). During the respiratory cycle, the flail segment moves paradoxically which can result in ventilatory compromise. Fractures of the thoracic spine account for $15-30 \%$ of all spinal fractures, with the most vulnerable segment being the thoracolumbar junction ( $\mathrm{T} 9$ to T12) region. More than two thirds of thoracic spine fractures are demonstrated by radiography, with almost all visible at CT. CT has the added advantage of demonstrating displacement or retropulsion of bone fragments into the spinal canal and impingement upon the spinal cord. 


\section{Key Point}

- Fractures of the sternum are extremely difficult to see on radiographs, and CT with contrast is indicated to evaluate for aortic and vascular injury, if sternal fracture is suspected.

\subsection{Thalassaemia}

With chronic hemolytic anemias (most commonly thalassaemia), hematopoietic tissues outside the bone marrow produce blood (extramedullary hematopoiesis). Normal marrow tissue expands outside the medulla through permeative erosions or by reactivation of previously dormant hematopoietic tissues [13]. Erythropoiesis is ineffective, leading to expansion of bone marrow space in the vertebral column, ribs, long bones, skull, and characteristically the facial bones (frontal bossing). Paravertebral and rib lesions are most often incidental but can occasionally cause cord compression due to mass effect on the spinal canal. On chest radiographs, thalassemia can manifest as bilateral lobulated paravertebral masses with rib expansion.

\section{Key Point}

- Chest radiography still plays a key role in the evaluation of the chest wall, particularly for calcification and air but also to assess the skeletal structures.

\subsection{Pleural Disease}

When assessing for pleural effusion or pneumothorax on radiography, one must keep in mind the patient's positioning (whether supine, semi upright, or upright), as air will rise upward and fluid will gravitate downward within the pleural space and influence the appearances. In critically ill supine patients, air will rise to lie in the anterior inferior costophrenic sulcus, leading to the deep sulcus sign with radiolucency inferior and laterally. The ipsilateral hemidiaphragm may also be more sharply delineated due to the air above it. Conversely, pleural fluid will layer posteriorly and can "cap" over the lung apex. On upright radiographs, intraperitoneal "free" gas will rise to the highest point, under the diaphragmatic domes, if sufficient time is allowed. Alternatively, ill patients can be rolled onto their side for decubitus views to diagnose pneumothorax or effusions. For suspected pneumothorax, the abnormal side should be put up to allow any air to rise, whereas to evaluate effusions, the side should down to get fluid to layer along the inner ribs.

\subsection{Pleural Effusion}

On upright radiographs, free-flowing pleural fluid displaces the lung away from the costophrenic sulci and blunts the angle, producing a fluid meniscus sign. With small pleural effusions, on upright lateral chest radiographs, one needs to check the posterior costophrenic sulcus (the lowest point) to look for dependent fluid. On the frontal view, view the lateral costophrenic angle or sulcus but bear in mind that subpulmonic pleural fluid can be present without blunting the lateral costophrenic angle. With a subpulmonic effusion, the ipsilateral "hemidiaphragm" will be of uniform attenuation, with a more lateral position of the apparent dome of the hemidiaphragm (with a gradual sloping medial aspect and a more sharply sloping lateral aspect). On supine radiographs, a fluid meniscus or interface (with lung or normal pleura) may not be seen if free-flowing pleural fluid layers posteriorly. With large pleural effusions, in supine patients, an apical pleural cap may be visualized as fluid layers around the apex of the lung.

If there are fibrinous adhesions or scars, pleural effusions may become loculated into localized components. Pleural fluid tracking into fissures can have a tapering cigar-shaped appearance. If pleural fluid in a fissure has a more rounded shape, it can look mass-like giving a "pseudotumor" appearance. Clues to its nature include the "mass" being homogenous in density, with a smooth outline, and having a different shape on two orthogonal projections. One can also review prior radiographs and see a decrease in size or resolution on follow-up.

When pleural effusions are large, they can deviate the mediastinal structures toward the opposite side. A rare but serious complication of large pleural effusions is diaphragmatic inversion. With diaphragmatic inversion, during respiration, the two hemidiaphragms move in opposite directions. This causes air to move over and back between the two lungs (pendulum respiration), resulting in increased dead space and significant respiratory compromise. Symptoms can be relieved with thoracocentesis which restores the affected hemidiaphragm to its normal position. It is easier to diagnose left hemidiaphragm inversion, as this causes mass effect upon the left upper quadrant, displacing the stomach air bubble inferiorly.

In hospitalized patients, pleural effusions are most commonly related to congestive cardiac failure or postsurgical. With congestive heart failure, the effusions are usually relatively symmetric bilaterally but occasionally more asymmetric (right larger than left) or unilateral. When effusions are unilateral or asymmetric with the left larger than the right in nonsurgical patients, other causes should be considered including pneumonia, infarction, neoplasm, trauma, and connective tissue disorders (especially systemic lupus 
erythematosus and rheumatoid arthritis). In acute pulmonary embolus (PE), small unilateral or bilateral hemorrhagic pleural effusions may be found.

Upper abdominal pathologies can cause sympathetic pleural effusions, usually unilateral. These include subdiaphragmatic abscess, pancreatitis, splenic trauma, and ascites. Clinical history will help, and thoracentesis can help distinguish some entities, for example, elevated amylase with pancreatitis. With ascites, due to hepatic failure, bilateral pleural effusions may develop from leak of fluid through diaphragmatic defects or with third spacing from hypoalbuminemia. With suspected right subdiaphragmatic fluid collections, ultrasound can be very helpful, but for suspected left upper quadrant abscess, CT is better.

Trauma, as the etiology of unilateral pleural effusion, may be apparent when there are multiple rib fractures but less obvious when due to vascular damage in the absence of fractures. Another less apparent cause might include incorrectly placed enteric tubes or vascular catheters. Accidental feeding tube placement into the lung may be uneventful as long as the malposition is detected before feeding is commenced. Malpositioned tubes in the pleural space are usually symptomatic due to complications including tension pneumothorax and empyema.

\section{Key Point}

- Patient positioning for radiography affects the appearances of pleural fluid with fluid layering dependently and posteriorly in the supine position, and if there is sufficient fluid, an apical pleural cap may be seen.

\subsection{Empyema}

Patients with pneumonia commonly have small sympathetic pleural effusions. Infection of the pleural space (empyema) can occur as a complication of pneumonia, with infections such as Mycobacterium tuberculosis and fungi, known for involving the pleural space. Differentiating empyema from lung abscess on chest radiographs can be performed by evaluating the shape of the collection on orthogonal views. A lung abscess tends to make acute angles with the adjacent chest wall, while empyemas will make a more obtuse angle. Furthermore, lung abscesses are round and of similar appearances and size on different projections in contrast to empyemas, which tend to be oval or linear on one projection and more spherical on the orthogonal projection. On contrastenhanced CT, empyema will have a space-occupying effect, deviating nearby bronchi and vessels, while bronchi and vessels will appear to enter and be obliterated by the lung abscess. The empyema will tend to have smoother margins than lung abscess which will be more ill-defined due to surrounding consolidation. In empyema, the "split pleura sign" with separation of the thickened visceral and parietal pleura may also be seen.

The presence of infection in the pleural space cannot be confirmed with imaging alone, and clinical (and laboratory) information are needed to support the diagnosis of infection. Imaging's role is to localize the source of infection to the pleural space, with the cause of pleural effusion often suggested based on clinical information. Early on, an empyema may be difficult to differentiate from a loculated pleural effusion. In some cases, thoracentesis and analysis of pleural fluid will be needed for diagnosis. Thoracic wall ultrasound can be helpful to guide thoracentesis.

\subsection{Pneumothorax}

Air can enter the pleural space in up to $40 \%$ of patients with acute chest trauma as a result of lung, tracheobronchial, or esophageal injury, and pneumothorax may be seen. To detect pneumothorax, upright frontal radiography is indicated as air tends to rise within the pleural space. However, if upright radiography is not feasible, lateral decubitus radiography (with the side of suspected pneumothorax up) is an alternative option. Computed tomography can identify many small and moderate-sized pneumothoraces which may not be visible on supine radiography.

Pneumothorax is best diagnosed by visualization of the visceral pleura outlined by air in the pleural space. Lucency and absence of vessels are less reliable signs which can be seen with presence of focal emphysema or bullae. As is the case with pleural effusions, detection of pneumothorax becomes difficult in the supine position, with the nondependent portion of the pleural space being at the anterior inferior costophrenic recess. Look for the deep sulcus sign, with lucency over the lower lateral chest and a sharply defined ipsilateral hemidiaphragm. A "too easily seen" sharply defined hemidiaphragm or increased lucency over the lung bases should raise concern for basilar pneumothorax, even without seeing the pleural line. A further sign is the "deep sulcus sign" corresponding to a sharply demarcated lateral sulcus. Pneumothorax can track into the fissures with air in the minor fissure a reported sign of supine pneumothorax.

Tension pneumothorax is a medical emergency, due to compression of mediastinal structures resulting in decreased venous return to the heart and hemodynamic collapse. As intrapleural pressure increases, the ipsilateral hemidiaphragm is depressed, with deviation of the mediastinal structures contralaterally. 
Pneumothorax is most often due to trauma or iatrogenic (with surgery or other interventions), but they can also occur secondary to ruptured blebs, and with interstitial lung diseases, where honeycomb change or cysts increase the risk of pneumothorax (e.g., in lymphangiomyomatosis or eosinophilic granuloma).

Other causes of pneumothorax, with an apparently normal CXR, include cavitary lesions in a subpleural location, for example, metastases or tuberculosis. Cavitary metastases include mostly squamous primary neoplasms and sarcomas. Increased intrathoracic pressure can potentially cause pneumothorax with an otherwise normal or nearly normal CXR, as can occur in asthma and pregnancy. Bronchial injury or bronchopleural fistula should be suspected when there is persistent or increasing pneumothorax with a functioning chest tube in place or the tube is on suction.

Pneumothorax ex vacuo is a complication of lobar collapse, with a sudden increase in negative intrapleural pressure surrounding the collapsed lobe [14]. This results in gas from the ambient tissues and blood being drawn into the pleural space with the seal between the visceral and parietal pleura of the adjacent lobe or lobes remaining intact. Once the bronchial obstruction is relieved and the collapsed lobe re-expands, the pneumothorax will resolve. It is critical to recognize this entity, as treatment is to relieve the bronchial obstruction rather than to insert a pleural drainage catheter.

\section{Key Point}

- In the supine position, a pleural edge may not be seen with pneumothorax, and the radiologist may have to rely on secondary signs including the deep sulcus sign or a hemidiaphragm that is too clearly defined.

\subsection{Hemothorax}

Hemothorax resulting from injury to large central pulmonary vessels, systemic thoracic veins or arteries, or lacerated viscera can be catastrophic with hemodynamic collapse, requiring urgent surgical intervention, whereas bleeding from low-pressure pulmonary vessels is usually self-limited. Like pleural effusion, hemothorax may be difficult to detect in the supine position, due to layering posteriorly. On supine radiographs, signs of hemothorax include an apical pleural cap, hazy increased opacity projected over the hemithorax, and confluent lateral pleural opacification. Whenever rib fractures are found, a hemothorax should always be considered, and $\mathrm{CT}$ is the most accurate way to detect tiny amounts of pleural fluid. A hemothorax can be suggested by CT, when the pleural fluid attenuation is $>35$ to $40 \mathrm{HU}$, and dependent layering of the higher attenuation hematocrit elements may be visible with enough blood in the pleural space. Consider a chylous pleural effusion when there is delayed or late appearance of pleural fluid that continues to slowly accumulate over days, particularly with penetrating injuries or following thoracic surgery, secondary to thoracic duct disruption.

\subsection{Pleural Plaques or Thickening}

With repeated asbestos exposure, bilateral pleural plaques occur commonly and sometimes visibly calcify. They are typically located at the hemidiaphragmatic domes and beside the posterolateral lower ribs [15]. When calcified plaques are viewed en face, they appear as irregularly shaped, poorly marginated opacities, sometimes with scalloped outlines giving a holly leaf-like appearance. A clue to the extrapulmonary origin of pleural plaques is that their long axis doesn't run parallel to that of the underlying pulmonary vasculature and bronchi.

Unilateral pleural plaques can be seen with asbestos exposure but also occur with previous hemothorax in trauma or following empyema. A small hemithorax with extensive pleural calcification (fibrothorax) may be seen after tuberculosis and untreated hemothorax. Non-calcified pleural plaques (and pleural implants) are often better demonstrated with CT rather than by radiography, in contrast to pleural effusion or pneumothorax.

\subsection{Pleural Implants and Masses}

While pleural masses may be seen with metastatic disease, pleural effusions more commonly occur. Many pleural implants are obscured on chest radiography (and even on CT) by surrounding pleural fluid. Some pleural masses, such as malignant thymoma, cause unilateral multifocal pleural implants without accompanying pleural fluid. On contrastenhanced CT, the higher attenuation soft tissue implants may be visualized, suggesting the malignant nature of the effusion. Intravenous contrast enhancement (and narrow soft tissue window widths) help distinguish the solid elements from pleural fluid. Dependent layering of hematocrit components with chronic pleural hematoma may look similar to malignant effusion and implants on CT.

Benign mesothelioma (benign fibrous tumor of the pleura) is often a single pleural mass, which ranges in size from small to very large [16]. These masses may be pedunculated and change in position and appearances by twisting around their pedicle. Large benign fibrous tumors are generally distinguishable from malignant mesothelioma due to their focal nature, with malignant mesothelioma being typically more widespread. Benign mesotheliomas are generally recogniz- 


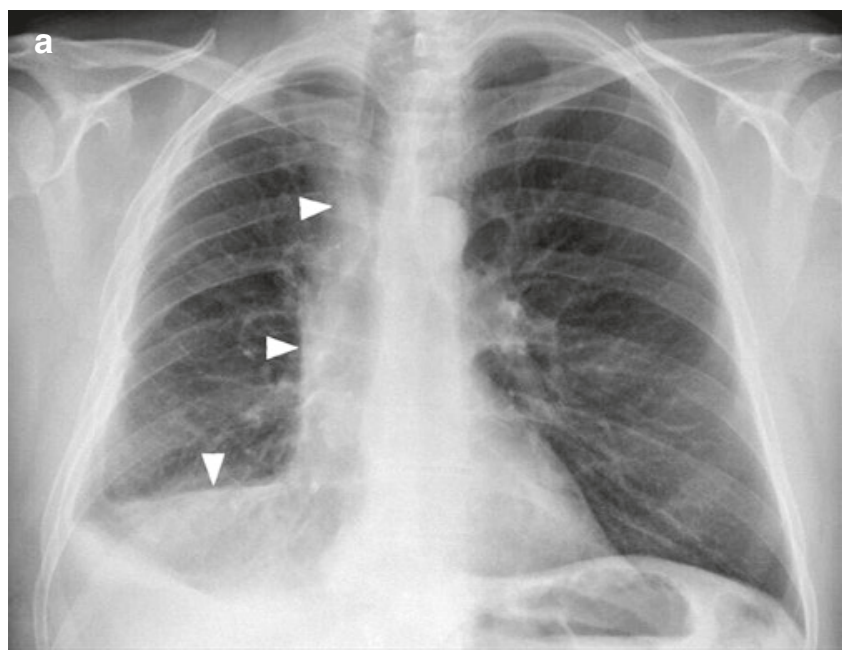

Fig.9.2 (a, b): A 65-year-old man with progressive shortness of breath on exertion and a dry cough. Frontal chest radiography (a) demonstrates a smaller right hemithorax with circumferential pleural thickening, including the mediastinal or medial aspect (white arrowheads).

able as extra-parenchymal pleural lesions due to their shape, with a broad based ill-defined pleural base, making obtuse angles to the lung. Difficulty arises when they occur in the fissures, appearing rounded and more sharply defined, raising suspicion for malignancy. Multiplanar imaging with CT localizes the "mass" to the fissure, making benign fibrous tumor a more likely diagnosis. Clinical associations can sometimes help in distinguishing benign fibrous tumor, such as associated hypertrophic pulmonary osteoarthropathy, and sometimes hypoglycemia. Benign mesotheliomas undergo malignant degeneration in up to a third of cases, and therefore management includes imaging workup prior to excision.

Pleural metastases are much more common than primary pleural neoplasms. Many cancers metastasize to the pleura with those in close proximity most likely, including the lung and breast. When a pleural mass is found and there is primary neoplasm elsewhere, metastatic disease should still be considered even if the primary malignancy isn't one frequently associated with pleural spread.

\section{Key Point}

- Pleural implants are more commonly caused by metastatic disease, with the main primaries being the lung, breast, mesothelioma and thymoma locally, and ovarian cancer.

Malignant mesothelioma is the most common primary tumor associated with pleural effusion. Lobulated pleural masses occur along with effusions, often unilateral. Previous asbestos exposure (or bilateral calcified pleural plaques) is a

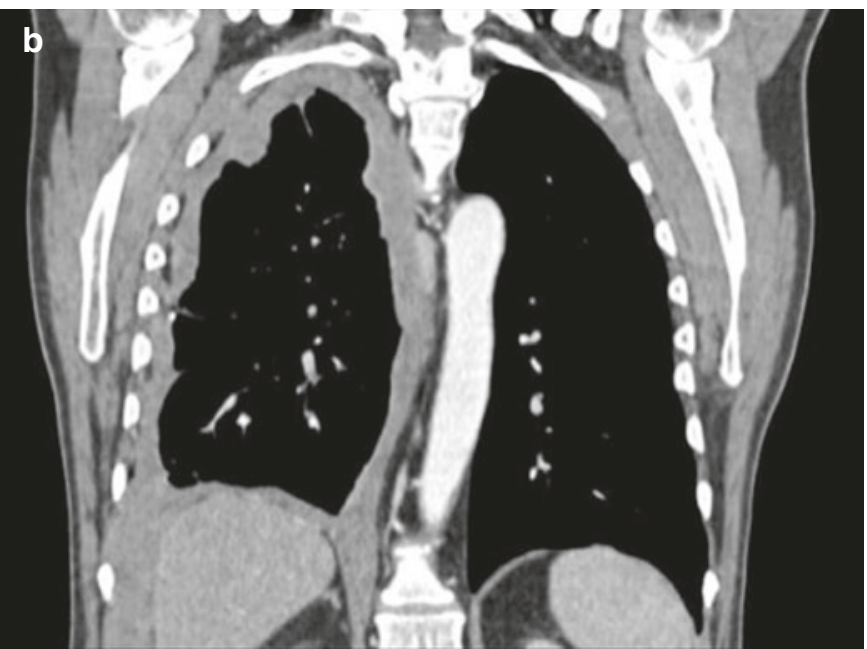

Coronal contrast-enhanced CT (b) shows nodular pleural thickening over the hemidiaphragmatic and mediastinal aspects, with a decrease in volume of the right hemithorax

helpful clue, but note that asbestos exposure is only documented in about half of malignant mesothelioma patients. Malignant mesothelioma can be differentiated from benign plaques by the involvement of the mediastinal pleura and the presence of pleural effusion. In some patients, a lack of mediastinal shift away from the affected side of and a decrease in volume in the presence of extensive pleural abnormality are clues to malignant mesothelioma [17, 18]. A differential to consider with circumferential nodular pleural masses often including the medial pleura are encountered is metastatic adenocarcinoma (Fig. 9.2).

Peritoneal metastases often occur with ovarian cancer, and malignant pleural effusions can occur when malignant seedlings pass through congenital hemidiaphragm defects. Massive malignant pleural effusions in ovarian cancer are known as Meigs syndrome.

\section{Key Point}

- Consider malignant mesothelioma or metastatic adenocarcinoma when lobulated pleural masses involving the mediastinal surface of the pleura are found.

\subsection{Diaphragmatic Disease}

On posteroanterior radiographs, the upper border of the right hemidiaphragm usually lies between the level of the fifth rib anteriorly and the anterior sixth-seventh rib interspace, typically a rib interspace higher than the left hemidiaphragm. On radiography, the diaphragm is inseparable from the abdominal structures below. On CT, the diaphragm is a thin 
soft-tissue attenuation linear structure which is thinner (and sometimes not clearly visible) toward the central tendon in contrast to the edges [19]. Muscle slips run obliquely along the inferior surface and may be asymmetric, with a mass-like appearance sometimes seen on cross section, especially in males, as they are more prominent. Prominent muscle slips with the muscle bulging upward on either side of the slips can give a scalloped appearance to the diaphragm, a normal variant. Multiplanar CT is useful to see the diaphragm due to the differences in attenuation of the diaphragm, adjacent intraperitoneal fat, nearby extrapulmonary fat, and aerated lung. Diaphragmatic defects (eventration) and hernias (Bochdalek posteriorly, Morgagni anteromedially) may be congenital or acquired (hiatus hernia, trauma, paresis, or paralysis). Predisposing conditions for all abdominal hernias include pregnancy, trauma, obesity, chronic constipation, and chronic cough.

\subsection{Bochdalek Hernia}

In adult patients, $90 \%$ of congenital diaphragmatic hernias found are Bochdalek hernias, more commonly left sided, possibly because of the protective effect of the liver on the right side [20]. Bochdalek hernias occur posterolaterally, through which intraperitoneal fat and, occasionally, solid organs such as kidneys or bowel loops pass through gaps between the costal and vertebral portions of the diaphragm [21]. Neonates and infants can present with respiratory compromise, with older children or adults sometimes presenting with acute or chronic gastrointestinal symptoms. Rarely, Bochdalek hernias can present as an acute abdomen due to complications of bowel incarceration, strangulation, perforation, or shock. With widespread CT imaging, most are discovered incidentally nowadays, as small defects, sometimes bilateral, particularly in patients with chronic obstructive airways disease. In these incidentally discovered Bochdalek hernias, no further action is needed. On CT imaging, discontinuity of the diaphragm posteriorly or posteromedially is usually easily seen with protrusion of peritoneal or retroperitoneal fat through the defect [21]. Less commonly, kidney, colon, small bowel, liver, or spleen may also herniate through a Bochdalek hernia. On chest radiography, the differential diagnosis for a contour deformity in the posterior hemidiaphragm includes lipomas, lung or diaphragmatic masses, neurogenic tumors, pulmonary sequestrations, or intrathoracic kidneys.

\subsection{Morgagni Hernia}

In adults, less than $10 \%$ of congenital diaphragmatic hernias found are Morgagni hernias, found anteromedially, due to failure of fusion of the sternal and costal portions of the hemidiaphragm [20, 22]. Morgagni hernias are more common on the right, perhaps because of greater support provided by the pericardial attachments on the left. In adults, omentum is most commonly found, with bowel, stomach, or liver rarely passing through. On imaging, Morgagni hernias appear as low attenuation masses in the anterior cardiophrenic angle and can be difficult to differentiate from prominent epicardial fat pads or pericardial cysts on radiographs. Other differential diagnoses for masses in the anterior cardiophrenic region include lipoma, liposarcoma, thymoma, thymolipoma, or teratoma. Coronal plane reformatted CT or MR images can demonstrate displaced curvilinear omental vessels within the "mass" or coursing across the diaphragmatic defect, typical features of Morgagni hernia.

\subsection{Diaphragmatic Eventration}

Hemidiaphragmatic eventration occurs with congenital muscular aplasia or thinning of a portion of tendon, which bulges upward with abdominal contents, most commonly anteriorly, because of the pressure gradient between the peritoneum and the thorax [21]. Unlike true hernias, the chest and abdominal contents are still separated by a layer of pleura, diaphragmatic tendon or muscle, and peritoneum and are incidental findings [19]. Rare symptoms of eventration include dyspnea, tachypnea, recurrent pneumonia, and failure to thrive, and these cases can be treated with surgical plication (tucking or folding and suturing). Hemidiaphragm eventration is nearly always found on the right side anteromedially, and with increasing intra-abdominal pressure (such as from increased abdominal obesity), it can become more pronounced. On imaging in eventration, there is elevation of the anterior portion of the hemidiaphragm, with a normal posterior origin (takeoff point) and position. This differs from diaphragmatic paresis or paralysis, where the entire hemidiaphragm will be elevated, including the posterior portion and origin (takeoff point). Relative thickening of the muscle at the edge of the eventration may be seen or sometimes undercut edges with ballooning upward of the eventrated portion on CT [21].

\subsection{Esophageal hiatus hernia}

The esophageal hiatus lies at the level of the tenth thoracic vertebral body and conveys the esophagus, sympathetic nerve branches, and the vagus nerve [20]. This hiatus functions as an anatomic sphincter by constricting on inspiration and preventing gastroesophageal reflux. The esophageal hiatus can enlarge with increasing age, abdominal obesity, and with general weakening of the musculofascial structures, leading to hiatal hernia. Herniation of 
part of the stomach most commonly occurs, with occasionally other abdominal organs, bowel loops or fat, or, rarely, the entire stomach. Intrathoracic hiatal hernias are most commonly sliding (in $90 \%$ of cases), with displacement of the gastroesophageal junction and a portion of (or entire) stomach upward into the thoracic cavity and loss of the usual antireflux mechanism. Less commonly, intrathoracic hiatal hernias are the rolling (paraesophageal) type, with a portion of (or all) the stomach or other abdominal contents, such as spleen or intestines rolling up above the gastroesophageal junction to enter the thoracic cavity. A combination of the two types (sliding and rolling) can also occur. On chest radiography, herniation of stomach will be visible as opacification behind the heart on the lateral view and obliteration of the azygoesophageal line on a frontal view [23]. On CT imaging, one can evaluate the location of the stomach and intra-abdominal organs, bowel loops, and peritoneal fat.

\subsection{Blunt Traumatic Diaphragmatic Rupture}

In about $5 \%$ of patients with blunt trauma, diaphragmatic rupture occurs, with most associated with significant intraabdominal injuries [24]. Injury mechanisms include frontal impact, causing increased intra-abdominal pressure and "blowing out" of the diaphragm, and lateral impact distorting the chest wall, causing shearing forces across the diaphragm. Diaphragmatic ruptures are thought to occur with equal frequency bilaterally, with ruptures on the right being clinically more occult due to protection from the liver. Consequently, left ruptures are reported in most (75$90 \%$ ) cases, with most tears posterolaterally at the weakest portion, the musculotendinous junction. After blunt trauma, most diaphragmatic tears are longer than $2 \mathrm{~cm}$ and many left tears even more than $10 \mathrm{~cm}$ in length.

About half of diaphragmatic ruptures are complicated by visceral organ herniation with the stomach and colon most commonly herniating on the left, and occasionally small bowel, spleen and kidney. The liver most commonly herniates on the right and sometimes colon. Herniated organs can strangulate, leading to ischemia, infarction, or obstruction. Sometimes, herniation after diaphragmatic rupture is delayed, with the relatively increased intraabdominal pressure gradually causing the defect to increase in size, increasing the likelihood of herniation of intraabdominal contents into the thorax. The increase in size of the diaphragmatic defect may be slowed down or prevented if patients receive positive pressure ventilation after their injury as this eliminates or reverses the pressure gradient. The time delay between injury and herniation is known as the latent phase and last for months to years, but most cases of strangulation manifest clinically within 3 years of the trauma.

Secondary signs of herniation can be seen at radiography including apparent elevation of the hemidiaphragm; an altered, irregular, discontinuous, or obscured contour; mediastinal shift to the contralateral side; viscera containing air above the hemidiaphragm; and if present, the gastric tube tip may be elevated with a U-shaped configuration. Similar appearances can be seen with other entities, including atelectasis, lung contusion, posttraumatic lung cysts, pneumothorax, subpulmonic pleural effusion, hiatal hernia, and phrenic nerve paralysis. Diaphragmatic eventration can have similar appearances, and comparison with old studies may be helpful here.

Multiplanar CT is more sensitive and specific, with signs of diaphragmatic injury including focal hemidiaphragmatic discontinuity; difficulty seeing the hemidiaphragm; a large defect between the torn ends of the hemidiaphragm; thickening and curling at the torn ends (due to muscular contraction); herniation of intraperitoneal fat, omentum, bowel loops, or abdominal viscera; focal narrowing of an organ or loop of bowel at the muscular defect (the the dependent viscera sign sign and "the dependent viscera sign") (the unsupported herniated organs or bowel fall dependently, abutting the posterior ribs) (Fig. 9.3). Other accompanying signs include pneumothorax, hemothorax, pneumoperitoneum, and hemoperitoneum. Coronal T2-weighted MRI is an alternative modality that can be used to evaluate for diaphragmatic injuries in the non-acute setting.

\section{Key Point}

- Hemidiaphragmatic ruptures can go unnoticed initially, particularly in intensive care patients with positive end inspiratory pressure ventilation, due to equal intrathoracic and intra-abdominal pressures, but they usually progress later, due to the relatively increased intra-abdominal pressure, and therefore should be repaired.

\subsection{Penetrating Diaphragmatic Injury}

Penetrating injuries of the diaphragm are more common than blunt injuries. The site of injury depends on the location and trajectory of the penetrating object. Penetrating injuries tend to be smaller, related to the size of the penetrating object, with most less than $2 \mathrm{~cm}$ and many below $1 \mathrm{~cm}$. Visceral herniation is uncommon with smaller penetrating injuries. Penetrating injuries are usually diagnosed clinically relying on the entry site and direction of the wound. Exploratory 

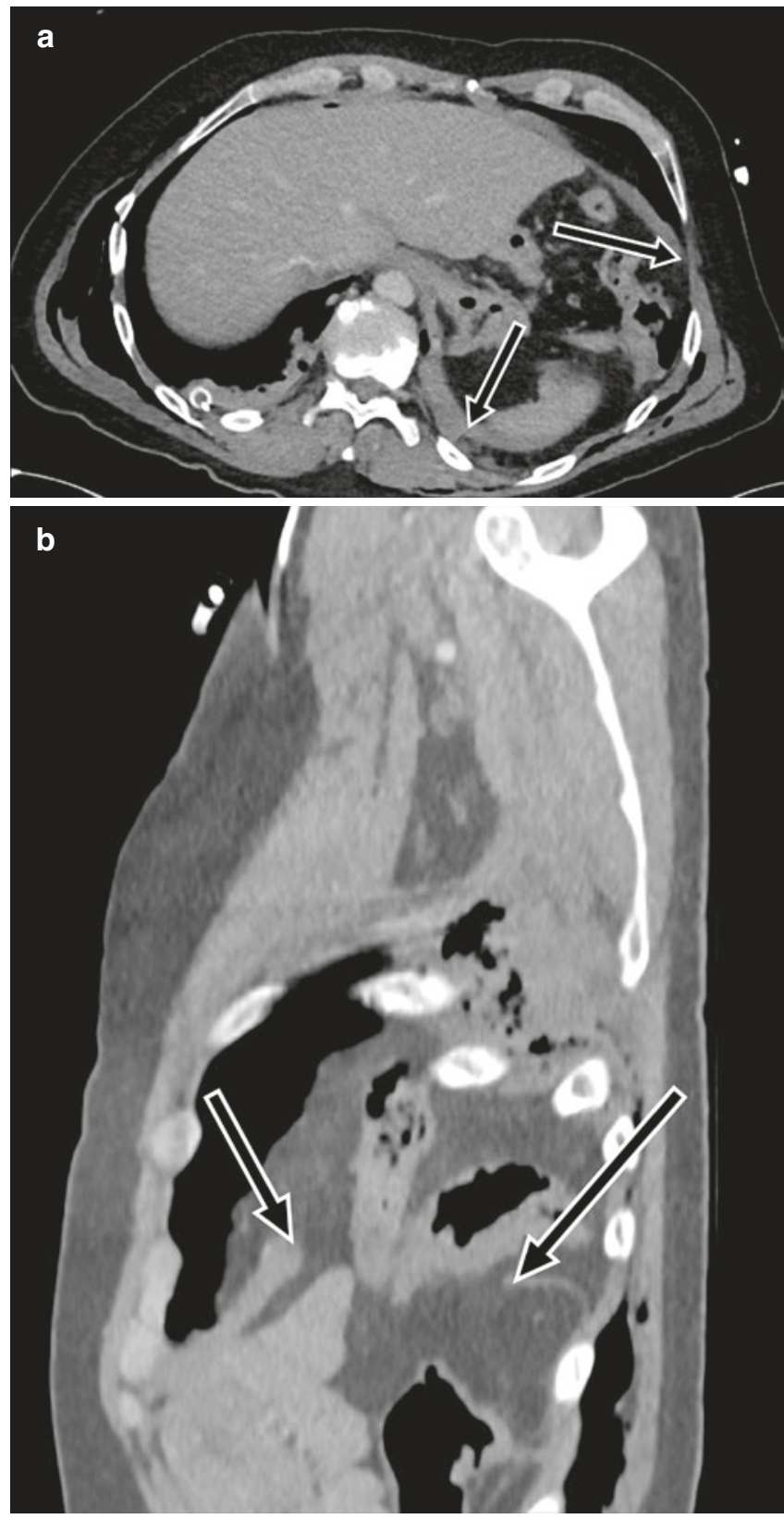

Fig. 9.3 (a, b): A 62-year-old woman who sustained blunt chest trauma in a road traffic accident. Axial contrast enhanced CT (a) shows diaphragmatic rupture, with thickened ends (black arrows) due to muscular contractions at the edge of the rupture. Note also the dependent location of the spleen (dependent viscera sign). There are also fractures of the right ribs and subcutaneous emphysema and a right basilar pleural drainage tube. Sagittal contrast-enhanced CT (b) demonstrates herniation of loops of colon through a large defect in the left hemidiaphragm, with thickening of the muscle at the margins (black arrows) of the defect, especially anteriorly

surgery is usually indicated in patients with penetrating injuries, at which time the diaphragmatic injury is diagnosed and repaired. CT imaging findings include pneumothorax, hemothorax, or radiopaque material associated with the projectile near the diaphragm or indicating the path through the diaphragm.

\subsection{Diaphragmatic Paresis or Paralysis}

Many processes result in diaphragmatic paresis or paralysis, including central nervous disease or local damage to the phrenic nerve from surgery or masses, but most cases are idiopathic, possibly caused by viral infection [25]. Thoracic imaging is usually unhelpful in determining etiologies for diaphragmatic paralysis, except in the case of direct invasion by intrathoracic tumors. Diaphragmatic paralysis is more commonly unilateral and often asymptomatic, being an incidental finding on imaging. Bilateral diaphragmatic paralysis is usually symptomatic, often presenting as respiratory compromise.

With bilateral diaphragmatic paralysis, frontal radiographs will demonstrate smooth elevation of the hemidiaphragms and decreased lung volumes and deep and narrow costophrenic and costovertebral recesses. The lateral view reveals a smooth contour and elevation of the entire diaphragm. Adjacent bibasilar linear atelectasis may be present. Apparent bilateral diaphragmatic elevation can also be seen with poor inspiratory effort, obesity, subpulmonic pleural effusions, subdiaphragmatic processes (like ascites, dilated bowel, and hepatomegaly), and pleural adhesions. With a unilateral elevated hemidiaphragm, the left may lie at a higher position compared to the right, or the right will lie more than one rib interspace higher than the left. A weak or paralyzed hemidiaphragm will show elevation in its entire course, including the posterior takeoff point, which helps differentiate it from hemidiaphragm eventration.

Fluoroscopic sniff testing can be helpful with unilateral diaphragmatic weakness or paralysis, by demonstrating slower downward (caudal) or paradoxical upward (with paralysis) movement during quiet inspiration, deep inspiration, and sniffing [25]. Sniff tests are not as useful for bilateral paralysis, may be falsely positive in normal individuals, and require user expertise. Ultrasonography can be used to diagnose paralysis, using the same principles as the fluoroscopic sniff test to assess diaphragmatic movement, but it requires operator experience. Chest and abdominal $\mathrm{CT}$ can be utilized to evaluate for processes that cause diaphragmatic elevation and simulate paralysis, including chronic obstructive airways disease, pleural effusions, subdiaphragmatic abscess, ascites, organomegaly, or ileus. Coronal (or sagittal) plane dynamic T2-weighted MRI imaging can be used to assess for paralysis (in centers with experience) with the diaphragm seen as a low T2 signal structure. Quantitative evaluation, including excursion, synchronicity, and velocity of diaphragmatic motion can also be performed with MRI.

\subsection{Tumors of the Diaphragm}

Primary tumors are rare, with more than half being benign, and the most common are lipomas [26]. Benign tumors are resected if there is diagnostic uncertainty or if they cause 
symptoms [26, 27]. Primary malignant lesions include rhabdomyosarcoma, followed by fibrosarcoma [2]. Secondary malignant lesions spread to the diaphragm by direct involvement from lung cancer, and other intrathoracic (mesothelioma, esophageal cancer) or intra-abdominal tumors (hepatic and adrenal cancer) more commonly occur. With malignant thymoma, drop metastases via the pleural space to the diaphragm can also occur as can intraperitoneal or serosal spread in ovarian cancer. Implants to the diaphragm can be found in benign processes such as endometriosis.

\subsection{Conclusion}

In evaluating the chest wall structures, the pleura, and the diaphragm, a combination of different imaging modalities can be employed, along with relevant clinical history (especially trauma and known primary malignancy). Previous imaging studies are particularly helpful in cases of suspected diaphragmatic trauma or paralysis. Comparing to the other (unaffected) side is helpful for chest wall pathology. Being familiar with image study techniques, coupled with knowledge of physics principles (air, water, blood, and soft tissue), can give valuable diagnostic clues.

\section{Take-Home Messages}

- Chest radiography has a major role in the evaluation of the chest wall, particularly for calcification and air but also to assess the skeletal structures.

- Certain fractures such as the sternum are extremely difficult to see on radiographs, and CT with contrast is indicated to evaluate for aortic and vascular injury, if sternal fracture is suspected.

- Remember the physical principles of fluid and air when assessing radiographs for pleural fluid or air. Pleural fluid will layer dependently in the supine position, posterior to the lungs. In the supine position, the pleural edge may not be seen with pneumothorax, and secondary signs, including the deep sulcus sign or a hemidiaphragm that is too clearly defined, should be looked for. Pleural implants are more commonly due to metastatic disease.

- Hemidiaphragmatic ruptures may not manifest initially, especially with positive end inspiratory pressure ventilation, which equalizes intrathoracic and intra-abdominal pressures, but defects will steadily progress after this, and therefore repair is indicated.

\section{References}

1. Hughes EK, James SL, Butt S, et al. Benign primary tumours of the ribs. Clin Radiol. 2006;61:314-22.

2. Tateishi U, Gladish GW, Kusumoto M, et al. Chest wall tumors: radiologic findings and pathologic correlation: part 2. Malignant tumors. Radiographics. 2003;23:1491-508.

3. Nam SJ, Kim S, Lim BJ, et al. Imaging of primary chest wall tumors with radiologic-pathologic correlation. Radiographics. 2011;31:749-70.

4. Carter BW, Benveniste MF, Betancourt SL, et al. Imaging evaluation of malignant chest wall neoplasms. Radiographics. 2016;36:1285-306.

5. Souza FF, de Angelo M, O'Regan K, et al. Malignant primary chest wall neoplasms: a pictorial review of imaging findings. Clin Imaging. 2013;37:8-17.

6. Bueno J, Lichtenberger JP 3rd, Rauch G, Carter BW. MR imaging of primary chest wall neoplasms. Top Magn Reson Imaging. 2018;27:83-93.

7. Schaub S, Sirkis HM, Kay J. Imaging for synovitis, acne, pustulosis, hyperostosis, and osteitis (SAPHO) syndrome. Rheum Dis Clin N Am. 2016;42:695-710.

8. Jeung MY, Gangi A, Gasser B, et al. Imaging of chest wall disorders. Radiographics. 1999;19:617-37.

9. Baez JC, Lee EY, Restrepo R, Eisenberg RL. Chest wall lesions in children. AJR Am J Roentgenol. 2013;200:W402-19.

10. Stewart VL, Herling P, Dalinka MK. Calcification in soft tissues. JAMA. 1983;250:78-81.

11. Donnelly LF. Use of three-dimensional reconstructed helical CT images in recognition and communication of chest wall anomalies in children. AJR Am J Roentgenol. 2001;177:441-5.

12. Miller LA. Chest wall, lung, and pleural space trauma. Radiol Clin North Am. 2006;44:213-24.

13. Orphanidou-Vlachou E, Tziakouri-Shiakalli C, Georgiades CS. Extramedullary hemopoiesis. Semin Ultrasound CT MR. 2014;35:255-62.

14. Woodring JH, Baker MD. Stark P (1996) Pneumothorax ex vacuo, Chest. 1996;110(4):1102-5.

15. Walker CM, Takasugi JE, Chung JH, et al. Tumor-like conditions of the pleura. Radiographics. 2012;32:971-85.

16. Rosado-de-Christenson ML, Abbott GF, McAdams HP, et al. From the archives of the AFIP: localized fibrous tumor of the pleura. Radiographics. 2003;23:759-83.

17. Wang ZJ, Reddy GP, Gotway MB, et al. Malignant pleural mesothelioma: evaluation with CT, MR imaging, and PET. Radiographics. 2004;24:105-19.

18. Truong MT, Viswanathan C, Godoy MB, et al. Malignant pleural mesothelioma: role of CT, MRI, and PET/CT in staging evaluation and treatment considerations. Semin Roentgenol. 2013;48:323-34.

19. Kharma N. Dysfunction of the diaphragm: imaging as a diagnostic tool. Curr Opin Pulm Med. 2013;19:394-8.

20. Nason LK, Walker CM, McNeeley MF, et al. Imaging of the diaphragm: anatomy and function. Radiographics. 2012;32:E51-70.

21. Sandstrom CK, Stern EJ. Diaphragmatic hernias: a spectrum of radiographic appearances. Curr Probl Diagn Radiol. 2011;40:95-115.

22. Taylor GA, Atalabi OM, Estroff JA. Imaging of congenital diaphragmatic hernias. Pediatr Radiol. 2009;39:1-16.

23. Anthes TB, Thoongsuwan N, Karmy-Jones R. Morgagni hernia: CT findings. Curr Probl Diagn Radiol. 2003;32:135-6.

24. Eren S, Ciriş F. Diaphragmatic hernia: diagnostic approaches with review of the literature. Eur J Radiol. 2005;54:448-59. 
25. Eren S, Kantarci M, Okur A. Imaging of diaphragmatic rupture after trauma. Clin Radiol. 2006;61:467-77.

26. Kim MP, Hofstetter WL. Tumors of the diaphragm. Thorac Surg Clin. 2009;19:521-9.
27. Tateishi U, Gladish GW, Kusumoto M, et al. Chest wall tumors: radiologic findings and pathologic correlation: part 1. Benign tumors. Radiographics. 2003;23:1477-90.

Open Access This chapter is licensed under the terms of the Creative Commons Attribution 4.0 International License (http://creativecommons. $\mathrm{org} /$ licenses/by/4.0/), which permits use, sharing, adaptation, distribution and reproduction in any medium or format, as long as you give appropriate credit to the original author(s) and the source, provide a link to the Creative Commons license and indicate if changes were made.

The images or other third party material in this chapter are included in the chapter's Creative Commons license, unless indicated otherwise in a credit line to the material. If material is not included in the chapter's Creative Commons license and your intended use is not permitted by statutory regulation or exceeds the permitted use, you will need to obtain permission directly from the copyright holder. 